\title{
Effects of Lower Limb Pattern Exercises on Balance and Gait in Patients with Hemiplegia after Stroke; a Single Case Report
}

\author{
Sang-Mi Chung OT, Ph.D ${ }^{1}$, Young-Dong Kim PT, Ph.D ${ }^{2}$, Woo-Nam Chang PT, Ph.D ${ }^{* 3}$ \\ ${ }^{1}$ Department of Occupatioal Therapy, Sanggi-Youngseo University, \\ ${ }^{2}$ Rehabilitation center, Hangagok rehabilitation hospital \\ ${ }^{* 3}$ Department of Physical Therapy, College of Health Welfare, Yong-In University
}

Purpose The aim of this study was to investigate effects of lower limb pattern exercises on balance and gait in patients with hemiplegia after a stroke. Methods A 70-year-old right-side hemiplegic patient after a stroke who was hospitalized in $\mathrm{H}$ hospital participated in this study. The intervention was applied 5 times a week for 3 weeks, 30 minutes per session, from October 10 to 31,2017 . Results The time period of the post10M Walk Test was the same as that of the pre-test, 89 seconds. 99 seconds was recorded at the baseline of the Timed Up and Go test (TUG) and 93 seconds shown at the post-TUG. The scores of Berg balance scale increased at the post-test from 19 to 27 points. Conclusion The intervention which was emphasized with dynamic stability and mobility of the affected foot at the beginning of the intervention with one leg stance exercise with dynamic control of the pelvis influenced the subject to achieve a more efficient and stable walking pattern.

Key words balance, gait, lower limb, pattern exercise, stroke

Corresponding author Woo-Nam Chang (bobathchang@hanmail.net)

Received date 18 January 2018

Revised date 23 January 2018

Accepted date 21 February 2018

\section{Introduction}

Since the majority of patients with strokes have a wide variety of impairments such as insufficient sensory, balance deficits, motor and cognition, etc., their activities of daily living become restricted. ${ }^{1)}$ Moreover, it is not uncommon for them to show an increase in postural perturbation with impaired balance and decreased abilities to shift body weight to the affected side, which can be causal factors in falls. ${ }^{2)}$ The purpose of assessing postural control and gait velocity are, in general, to evaluate progress. ${ }^{1)}$ The typical features of hemiplegic walk contain reduced gait velocity and asymmetry of joint angles in the lower limbs, step length, stance and swing phase time. Therefore, difficulty in balance with an increased probability of falling and restrictions on activities of daily living and taking part in activities in social communities are

doi : http:dx.doi.org/10.17817/2018.01.23.111234 developed. ${ }^{3)}$ Moreover, hemiplegic patients struggle to keep their center of mass (COM) in base of support (BOS) so that by using the pelvis moving backward during trunk movements alternatively, COM is maintained within BOS. Controlling COM to be in BOS can be achieved when the various sensory inputs coming from outside of the body are carried appropriately to each body segment. The feet play a significantly important role in terms of receiving the sensory inputs and maintaining balance. This is because the human body segments are interconnected from the bottom to the top. Consequently, a problem that happens in one segment would influence the other body parts' alignments. ${ }^{4)}$ Walking difficulty, which is one of the more significant problems patients with hemiplegia has, is presented due to weakness of the affected side of the body. "Therefore, the purpose of this study was to examine how to recover muscle weakness in the affected side of the body through sensory information given by a physical therapist, and was to 
clarify whether it can ultimately benefit balance and gait capacity.

\section{Materials and Methods}

\section{Participant}

This study was conducted with a 70 years old rightside hemiplegic patient after a stroke who was hospitalized in $\mathrm{H}$ hospital. The intervention was applied 5 times a week for 3 weeks, 30 minutes per session, from October 10-31, 2017. The subject provided her written informed consent to take part in this study prior to its commencement.

\section{Measurement tools and outcome measures \\ 1) Timed Up and Go test (TUG)}

This test is used to assess the fundamental mobility and balance quickly. The procedure is as follows: 1) a subject sits in an armchair, 2) stands up and walks forward 3 meters, 3) turns around at the turning spot, 4) walks back to the place the subject came from, 5) sits down in the armchair again. The meaning of taking above 30 seconds in this test is that the subject cannot do any activities alone due to a lack of basic mobility capacity. The intra-rater reliability was $r=.99$ and inter-rater reliability was $r=.98$.

\section{2) $10 \mathrm{M}$ Walk Test}

A subject walks 10 meters but should not stop at the spot 10 meters from the starting line. A tester measures the time right after the subject passes the line of 2 meters from the starting line and stop measuring at the line of 8 meters from the starting line. Therefore, the tester measures walking time of a total of 6 meters. ${ }^{7)}$

\section{3) Berg Balance Scale (BBS)}

BBS is a test used to assess balance quantitatively in the elderly and consists of 14 different categories including diverse postures and activities that a subject should complete within a limited time. The total score is 56 points. A score between 0 to 20 points stands for a severe balance disorder. A score between 21 to

40 points stands for a moderate balance disorder and a score between 41 to 56 points stands for a mild balance disorder. This test was created to assess balance for the elderly, but there is also use with high reliability in assessments for people with strokes. The intra-rater reliability was $\mathrm{r}=.98$ and inter-rater reliability was $r=.99 .^{8)}$

\section{Intervention}

The intervention for this study is as follows:

1) Make a subject lie down, face up

2) A patient occupies wide base of support in supine position

3) Hold one part of the affected foot with one hand and make the other part of the foot move with the other hand

4) Give diverse stimuli such as traction, compression, mobilization to get better mobility in each toe

5) Make the subject sit on at the edge of the plinth

6) Make sure the subject sits tight and comfortably

7) Make the pelvis move without any movement of other parts of the body

8) Guide the subject to stand up

9) Grasp the little toe with one hand to make it lift first to lead dorsiflexion and eversion of the foot

10) Make the non-affected foot move forward steadily to strengthen the affected foot in the stance phase

11) Move the non-affected foot to go back to the place where the foot came from

12) Repeat forward and backward movement of the non-affected foot several times

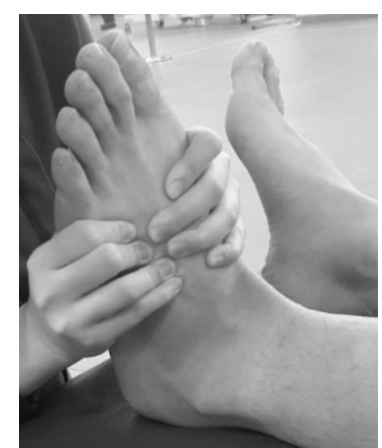

Figure 1. Facilitating intrinsic muscles

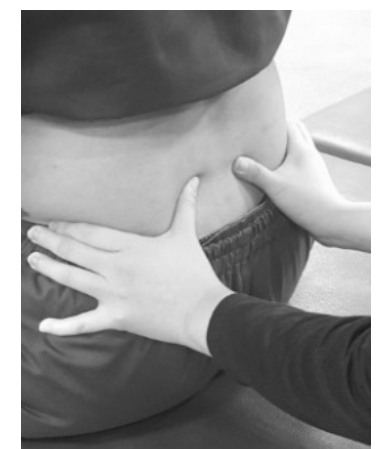

Figure 2. Pelvic movements 


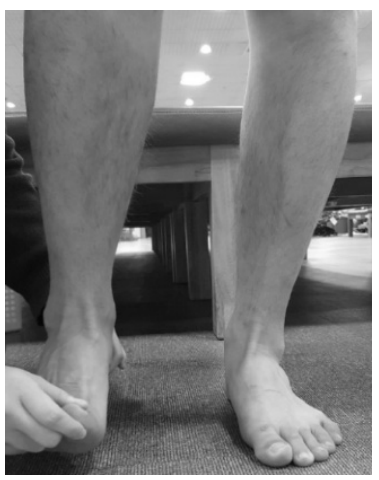

Figure 3. Initiation of the non-affected foot forward

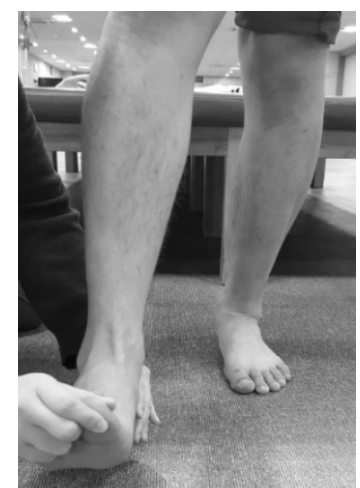

Figure 4. Heel contact on the floor

\section{Results}

After 3-week intervention period, the time period of post10M Walk Test was as same as that of pre-test which was 89 seconds. 99 seconds was recorded at the baseline of TUG and 93 seconds was shown at the post-TUG. The scores of BBS increased at the post-test from 19 to 27 points.

\section{Discussion}

Patients with hemiplegia have a high risk of falling and difficulty with activities of daily living, due to diminished muscle strength and balance. ${ }^{9)}$ It takes much more time and a longer route for patients with hemiplegia to reach at a certain point, such as when doing a reaching and grasping task. ${ }^{1)}$ Moreover, recovering balance and gait is vital for them to achieve a greater quality of life and reduce the potential cost for long term care. Therefore, through a proper and case-focused therapy, withdrawing plasticity is crucial in the damaged areas in the brain that will improve function. ${ }^{10)}$ Patients with hemiplegia with balance deficit show utmost worsening on gait parameters, such as a longer time and a greater number of steps when performing all task conditions ${ }^{11)}$ and use their hip to control balance, so-called hip strategy, instead of ankle strategy because the ground reaction force was changed which leads to the greater use of the hip flexors than the hip extensors. ${ }^{12)}$ The previous study found that 251 subjects who were inner-city-dwelling older adults aged 60 to 95 years had score of 53 (range from 29 to 56 ) on the BBS. ${ }^{13)}$ In this study, the subjects performed with better movement and maintaining postures after the intervention. This was because the intervention was focused on the sensory input at the beginning with diverse ways, resulting in better sensory adoption. Since movement control comes from better sensory information and conveying the information to the brain, the intervention ultimately benefits in the enhancement of the somatosensory system. In the previous study, there was a significant decrease in an experimental group after a stair-gait training. This is because dynamic balance ability was improved from the stair-gait training which was a walking in diversely different directions Besides, the stair-gait training had benefit on gait velocity in patients with hemiplegia from $0.44 \mathrm{~m} / \mathrm{s}$ at before -training to $0.75 \mathrm{~m} / \mathrm{s}$ at after-training ( $>$ >.05). ${ }^{9)}$ In this study, the subjects came to perform better walking skills, such as going forward and turning, as well as walking long distances in a reduced time after the intervention. This is because the subjects trained in a one-leg stance exercise, with better control of ankle and foot, resulting in a better gait cycle and symmetric gait, consequently leading to fast walking. Therefore, the intervention which was emphasized in dynamic stability and mobility of the affected foot at the beginning of the intervention with the one-leg stance exercise, with dynamic control of the pelvis, influenced the subjects to achieve a more efficient and stable walking pattern. Limitation of this study was that proper and objective assessment equipment was not used to get data. Future studies should be conducted with more computerized measurement tools to show objective data such as GAITRite to measure temporal and spatial parameters of the gait cycle or electromyography to measure muscle activity. However, because this was a single subject study, the intervention applied to the subject with hemiplegia was the main issue to be appealed in this study. 


\section{References}

1. van Dijk MM, Meyer S, Sandstad S, et al. A cross-sectional study comparing lateral and diagonal maximum weight shift in people with stroke and healthy controls and the correlation with balance, gait and fear of falling. PloS one. 2017;12(8).

2. Lopes PG, Lopes JA, Brito CM, et al. Relationships of balance, gait performance, and functional outcome in chronic stroke patients: A comparison of left and right lesions. BioMed Res Int. 2015.

3. Lee ME, Jo GY, Do HK, et al. Efficacy of aquatic treadmill training on gait symmetry and balance in subacute stroke patients. Ann Rehabil Med. 2017;41(3):376-86.

4. Kim YD, Lee KB, Roh HL. Immediate effects of the activation of the affected lower limb on the balance and trunk mobility of hemiplegic stroke patients. J phys ther sci. 2015;27(5):1555-7.

5. Lee DG, Jeong SK, Kim YD. Effects of underwater treadmill walking training on the peak torque of the knee in hemiplegic patients. J phys ther sci. 2015;27(9):2871-3.

6. Podsiadlo D, Richardson S. The timed "Up \& Go": a test of basic functional mobility for frail elderly persons. J Am Geriatr Soc. 1991;39(2):142-8.

7. Thompson $\mathrm{P}$, Beath $\mathrm{T}$, Bell $\mathrm{J}$, et al. Test retest reliability of the 10-metre fast walk test and 6-minute walk test in ambulatory school-aged children with cerebral palsy. Developmental Medicine \& Child Neurology. 2008;50(5):370-6.
8. Berg K, Wood-Dauphinee S, Williams J. The balance scale: reliability assessment with elderly residents and patients with an acute stroke. Scand J Rehabil Med. 1995;27(1):27-36.

9. Kim YH, Kim JH, Jeon HJ. The effects of task oriented stair gait training on muscle activities of the lower extremity and balance in stroke patients. J Korean soc neurother. 2018;22(1):22-30.

10. Maguire C, Sieben JM, Erzer F, et al. How to improve walking, balance and social participation following stroke: a comparison of the long term effects of two walking aids-canes and an orthosis TheraTogs-on the recovery of gait following acute stroke. A study protocol for a multi-centre, single blind, randomised control trial. BMC neurol. 2012;12:18.

11. Manaf H, Justine M, Omar M. Functional balance and motor impairment correlations with gait parameters during Timed Up and Go Test across three attentional loading conditions in stroke survivors. Stroke res treat. 2014.

12. Kim YD. Effects of modified bridging exercises on static postural control of a poststroke hemiplegic patient who had received surgery for lumbar spinal stenosis: a case report. J phys ther sci. 2015;27(4):1277-8.

13. Newton RA. Balance screening of an inner city older adult population. Arch phys med rehabil. 1997;78(6): $587-91$. 